Review

\title{
Review on Complete Mueller Matrix Optical Scanning Microscopy Imaging
}

\author{
Aymeric Le Gratiet ${ }^{1, *(0)}$, Ali Mohebi ${ }^{1,2}\left(\mathbb{D}\right.$, Fabio Callegari ${ }^{1,2}$, Paolo Bianchini ${ }^{1}$ and Alberto Diaspro ${ }^{1,2}(\mathbb{C}$ \\ 1 Nanoscopy and NIC@IIT, Istituto Italiano di Tecnologia, Via Morego 30, 16163 Genova, Italy; \\ ali.mohebi@iit.it (A.M.); fabio.callegari@iit.it (F.C.); paolo.bianchini@iit.it (P.B.); alberto.diaspro@iit.it (A.D.) \\ 2 Department of Physics, University of Genoa, Via Dodecaneso 33, 16146 Genova, Italy \\ * Correspondence: aymeric.legratiet@iit.it
}

check for

updates

Citation: Le Gratie, A.; Mohebi, A.; Callegar, F.; Bianchini, P.; Diaspro, A. Review on Complete Mueller Matrix Optical Scanning Microscopy Imaging. Appl. Sci. 2021, 11, 1632. https:// doi.org/10.3390/app11041632

Academic Editor: Stefano Selci

Received: 15 January 2021

Accepted: 5 February 2021

Published: 11 February 2021

Publisher's Note: MDPI stays neutral with regard to jurisdictional claims in published maps and institutional affiliations.

Copyright: (c) 2021 by the authors. Licensee MDPI, Basel, Switzerland. This article is an open access article distributed under the terms and conditions of the Creative Commons Attribution (CC BY) license (https:/ / creativecommons.org/licenses/by/ $4.0 /)$.

\begin{abstract}
Optical scanning microscopy techniques based on the polarization control of the light have the capability of providing non invasive label-free contrast. By comparing the polarization states of the excitation light with its transformation after interaction with the sample, the full optical properties can be summarized in a single $4 \times 4$ Mueller matrix. The main challenge of such a technique is to encode and decode the polarized light in an optimal way pixel-by-pixel and take into account the polarimetric artifacts from the optical devices composing the instrument in a rigorous calibration step. In this review, we describe the different approaches for implementing such a technique into an optical scanning microscope, that requires a high speed rate polarization control. Thus, we explore the recent advances in term of technology from the industrial to the medical applications.
\end{abstract}

Keywords: polarimetry; mueller matrix; optical scanning microscopy; imaging

\section{Introduction}

Polarization-based imaging techniques are powerful approaches having the unique ability to produce specific contrasts for revealing hidden information [1]. Over the last few decades, it has shown its efficiency in multiple areas showcasing its particular sensitivity to the medium structure and orientation [2]. As follows, it has been referred to a wide range of experimental applications from astrophysics [3,4], remote target detection [5-8], and micro/nanoparticles in a turbid or highly scattering medium [9-14]. Especially, Mueller matrix polarimetry is the most comprehensive method as it provides the full polarimetric response of a sample through its $4 \times 4$ Mueller matrix (MM). At least 16 intensity measurements, obtained by a set of 16 coding and decoding polarization states, must be performed to determine the 16 real independent element of the Mueller matrix $m_{i j}$ [15]. Various methods were then developed based on either temporal, spatial, or spectral polarization coding and decoding [16]. For imaging microscopy applications, two approaches provide the complete Mueller matrix elements $m_{i j}(\mathrm{x}, \mathrm{y})$ to be measured across a sample in two dimensions.

The first and most common approach incorporates the full-field imaging approach using a CCD or a CMOS camera including full-field polarization coding and decoding stages. Most wide-field Mueller imagers use dynamic polarization optics to modulate light polarization in the temporal domain. The methodology consists of a sequential acquisition of at least 16 polarization-resolved intensity images of the sample for reconstructing the complete Mueller matrix in just few seconds up to few minutes. For tracking any fast polarimetric changes, recent works have proposed a snapshot Mueller matrix microscope based on the simultaneous coding/decoding of the polarization states physically splitted in the plane of the sensor which is of great interest for biomedical diagnosis [17]. The idea is to couple a micro-polarizer array with a CCD or CMOS camera. Each pixel of the camera is linearly polarization encoded $\left(0^{\circ}, 45^{\circ}, 90^{\circ}\right.$ and $\left.135^{\circ}\right)$ and a group of four pixels gives a super pixel allowing the reconstruction of the four Stokes coefficients through a 
field-programmable gate array (FPGA) card in real-time [18]. Nowadays, since this passive technique does not require any sophisticated mathematical model or expansive optical features, it exists numerous commercial polarization-resolved camera, dedicated for remote sensing or widefield imaging [19-21]. However, the limitation of such a method is the need of taking into account the crosstalk between pixels and the loss of spatial resolution due to the definition of the super-pixels [22]. Another limitation is the difficulty to be implemented with other imaging modalities due to the space required for the polarimetric optical features.

In an attempt to preserve the axial optical resolution through the illumination volume, the second approach consists in point-by-point scanning laser microscopy (SLM) of the sample. Particularly, this review article is dedicated to the description of the experimental advances in the field of complete MM in optical scanning microscope for imaging pixelby-pixel the xy focal plane of the objective. This measurement method differs from using the scanning beam and is based on collecting the angular fingerprint of the sample as it is often proposed in polarization-resolved scatterometry [23]. In this review, we demonstrate the capability of this method for imaging localized contrasts based on the single optical and structural properties of the sample at the microscale. Commonly, the imaging of such small objects is made using fluorescence techniques showing an extraordinary capability of tracking localized molecules beyond the diffraction limit. Nevertheless, it requires the use of fluorophores and high light-dose that can alter the sample organization. For this reason, the main advantage of developing a full MM imaging system is that there is no labeling process or a priori knowledge of the sample. Thus, this technique is known to be a label-free method and the contrasts is based on the single interpretation of the light-matter interaction arisen from the sample optical fingerprint. We show that developing full MM in a scanning microscopy configuration offers the advantage to be easily implementing into commercial setups giving the capability toward multimodal imaging. We used databases such as PubMed and the Web of Science to gather information for this article. First, we discuss the different approaches of coding and decoding the polarization suitable with the scanning beam across the sample. Furthermore, we present different applications of this technique, demonstrated its performances from the earliest works to the most recent advances in the optical scanning microscopy field.

\section{Mueller Matrix Optical Scanning Architecture}

Any MM polarimeters are composed of two optical modules aiming to encode/decode the polarization states after interaction with the medium. First, the Polarization States Generator (PSG) encodes the polarization states from the incoming light source (emitted by a lamp or a laser diode), as described by the Stokes vector $\overrightarrow{S_{i n}}=\left[S_{0}, S_{1}, S_{2}, S_{3}\right]$, where $S_{0}$ is the total transmitted light, $S_{1}, S_{2}$ and $S_{3}$ are related to the linear horizontal/vertical, $+45^{\circ} /-45^{\circ}$ and circular right/left polarization states. Their combinations provide valuable information increasing the information available, such as the Degree Of Linear Polarization (DOLP), and Circular (DOCP) [24]. Then, after the sample, the transformation of the polarized light is decoded by the Polarization States Analyzer (PSA) giving the output Stokes vector as $\overrightarrow{S_{\text {out }}}=[S 0, S 1, S 2, S 3]^{\text {out }}$ and collected by a photodetector. In the literature, the mix of the PSA and the detector part is also referred to as the Polarization States Detector (PSD). The MM, noted M, describes how the polarization state of the input light changes upon interaction with the sample by

$$
\overrightarrow{S_{\text {out }}}=[M] \overrightarrow{S_{\text {in }}}
$$

where $\overrightarrow{S_{\text {in }}}$ and $\overrightarrow{S_{\text {out }}}$ are the input and output Stokes vectors. Since the imaging contrasts erase from the only linear optical response of the medium with the polarized light, there is no need of using high power laser source or extremely sensitive photodetectors, which decreased drastically the cost and the complexity of polarization-resolved techniques.

The main challenge for implementing this technique in an SLM configuration is to adapt a proper methodology for encoding/decoding the polarization states in a way 
to retrieve the full MM pixel-by-pixel. Additionally, the critical issue for extracting the accurate MM is the calibration of the full optical system. It has to count on the polarization state inaccuracy from the PSG and PSA errors combined with the polarimetric artifacts because of the multiple interaction with the optical features composing the microscope (lenses and mirrors). In SLM, the specimen is scanned by a diffraction limited spot of a laser light after reflection in galvanometric mirrors. Then, the light is transmitted or reflected by the in-focus illuminated volume element (voxel) of the specimen and is focused onto a photodetector. For MM SLM, the lateral resolution is approaching the diffraction limitation given by the objective numerical aperture express as

$$
R_{\text {lateral }}=\frac{\lambda}{2 \cdot N A}
$$

We present the different configuration available from such a technique Figure 1.



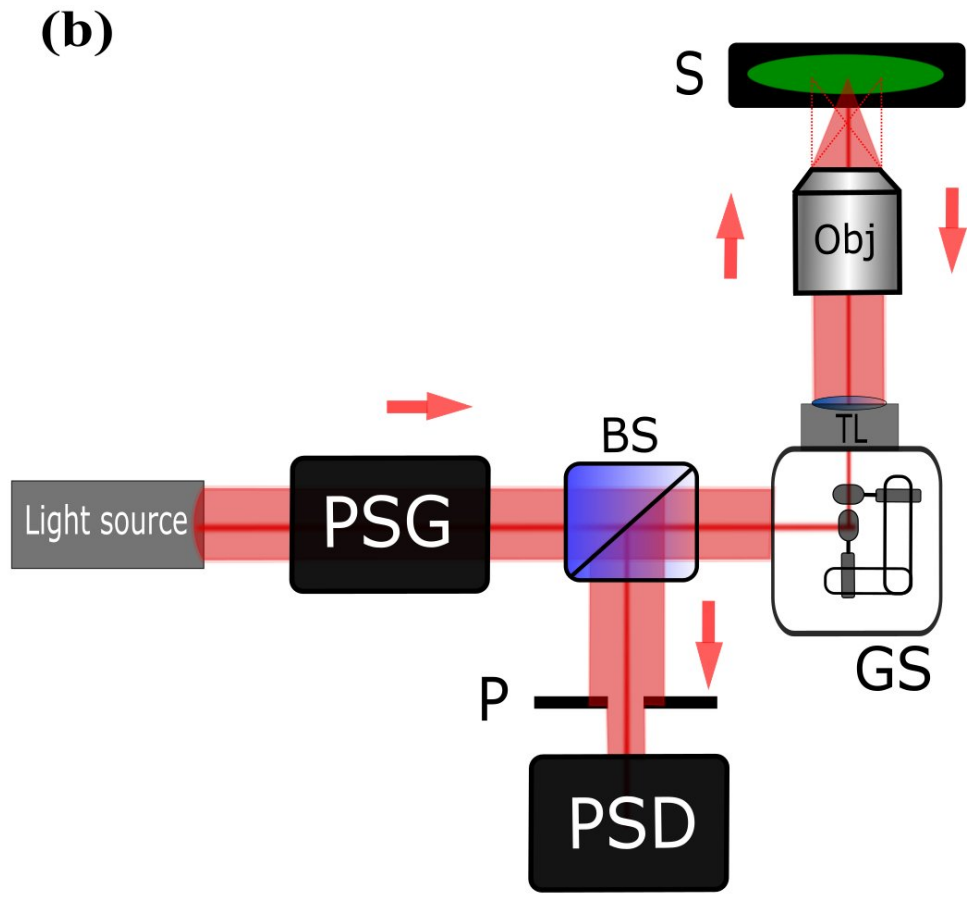

Figure 1. Block diagram for implementing a general Mueller matrix (MM )polarimeter into a scanning laser microscopy (SLM) architecture (a) in transmission and (b) in confocal reflection configuration. PSG: polarization states generator. Obj: microscope objective. GS: galvanometric scanner. TL: tube lens. S: sample. BS: beamsplitter. P: pinhole. PSD: Polarization States Detector.

Usually the calibration steps consist in measuring the polarimetric contributions independently of the sample of (1) the PSG and PSA blocks and (2) the optical microscope features fingerprints. First, the PSG and PSA could be calibrated separately using dedicated methods according to its architecture. Studying the conditioning of the measurement matrix have been used by several authors to optimize the polarimeter performance. Particularly, if a simple set of rotated polarizers and waveplate are used for encoding the polarization states, a simple model such as Eigenvalue Calibration Method (ECM) [25-27] have been proven to be robust and versatile enough. In this way, the trace of the matrices measured using simple reference sample at specific orientation gives a Condition Number (CN) that resumes the robustness against noise propagation through the whole system. If the PSG and PSA are more complex to model, for instance by using electro optics devices 
(Pockels cells or photoelastic modulators) [28], the system is considered as a "black box" evaluated by a figure of merit named Equally Weighted Variance (EWV) criterion where only the global experimental noise propagation is considered [29]. In a confocal mode for the reflection imaging configuration, the double pass through the sample has to be considered [30]. Indeed, it becomes even crucial for polarization tracking through the overall Point Spread Function (PSF) volume. For this reason, a variant method of the $\mathrm{ECM}$ has been introduced requiring the measurements of the polarimetric properties of reference samples such as linear polarizer or waveplate at specific orientation. However, in a confocal mode it is important to recalibrate the system for each pinhole size resulting in an increasing of the number of measurements. Additionally, it is important to assume that the light is reflected by a perfect mirror at normal incidence, and that the fingerprint of the polarization features is independent of the direction of propagation of the light. This final hypothesis is not easy to achieve experimentally limiting the use of such approach for versatile implementation. Second, the microscope body is composed of successive multiple optics (lenses, filters and mirrors) that transform completely the generated polarization states. Thus, the measurement pixel-by-pixel of its optical properties could be easily performed by removing any sample in transmission or by placing a simple reflective mirror in reflection. The double pass ECM method could be also used and provides simultaneously the Mueller matrices of the encoding/decoding blocks and of the microscope body [30]. Finally, the Mueller matrix of the sample is isolated by using matrices inversion based on Mueller/Stokes formalism, as follow

$$
\left[M_{\text {sample }}(x, y)\right]=\left[M_{\text {mes }}(x, y)\right] \cdot\left[M_{\text {microscope }}(x, y)\right]^{-1},
$$

where $\left[M_{\text {sample }}\right],\left[M_{\text {microscope }}\right]$ and $\left[M_{\text {mes }}\right]$ are the Mueller matrices of the sample, the microscope and the total (microscope+sample).

Most of the MM SLM microscope deals with slow polarization states encoding/ decoding rate, by means slower than the pixel dwell time achieved by the galvanometric scanner (GS). However, this limitation has been overcome by simply acquiring successive images with different polarization states. Most recent advances for getting the full MM images at the pixel dwell time rate have proposed a snapshot approach, inspired by Optical Coherence Tomography (OCT) setups able to get the MM in the faster way based on our knowledge. Although the full-field technique is potentially faster than the scanning technique, the latter opens the way for implementation of various imaging modalities (Mueller/confocal/nonlinear) on a same scanning microscope [31].

\subsection{Temporal Domain Encoding/Decoding}

In the 1970s/1980s, the very first development in polarization-resolved microscopy was of interest in the research community for its advantage of extracting the label-free contrasts. Due to the low technological requirement, the temporal control of the polarized light was the first approach for such instrumentation based on the use of rotating optical features or electro-optics modulators. In a single-point measurement, the technique was based on the differential polarized (linear or circular) intensity collection, leading to the determination of only few Mueller matrix elements [32-37]. Thus, the method has been deported into an SLM configuration for imaging high-ordered macromolecules and biopolymers, such as chromatin or blood cells [38-43]. The main motivation for such implementation was to substitute the fluorescence techniques by imaging and tracking very localized molecules based on the single optical response of the sample without sample preparation. Later, the first full MM polarimeter imaging associated to an SLM configuration have been proposed for ophthalmology, based on OCT imaging in 1999 [44]. It allows the imaging of the full $\mathrm{MM}$ in reflection configuration using LCVRs. Even if there is no need of any moving part, the technique consists in measuring 16 successive double-pass images with an exposure time of $4 \mathrm{~s}$, resulting in a full acquisition in one minute. Considering that this approach required a low power source for not damaging the retinal tissues, the method is coupled with an important post-data treatment for reducing the noise and increasing the Signal-to- 
Noise ratio (SNR). A block diagram resuming this technique in transmission configuration is proposed Figure 2.

(a)

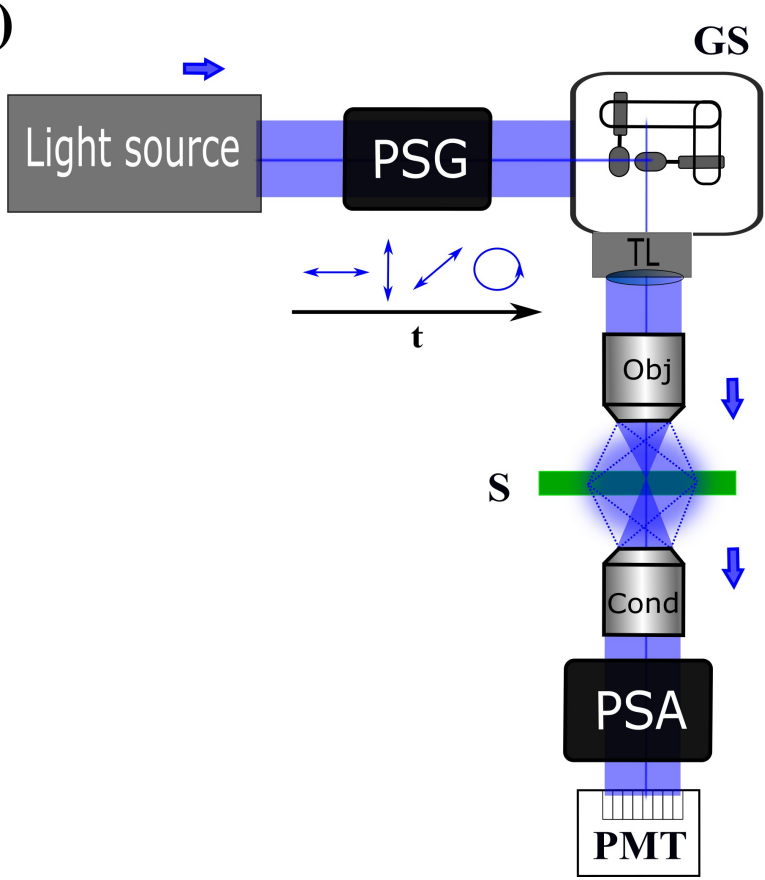

(b)

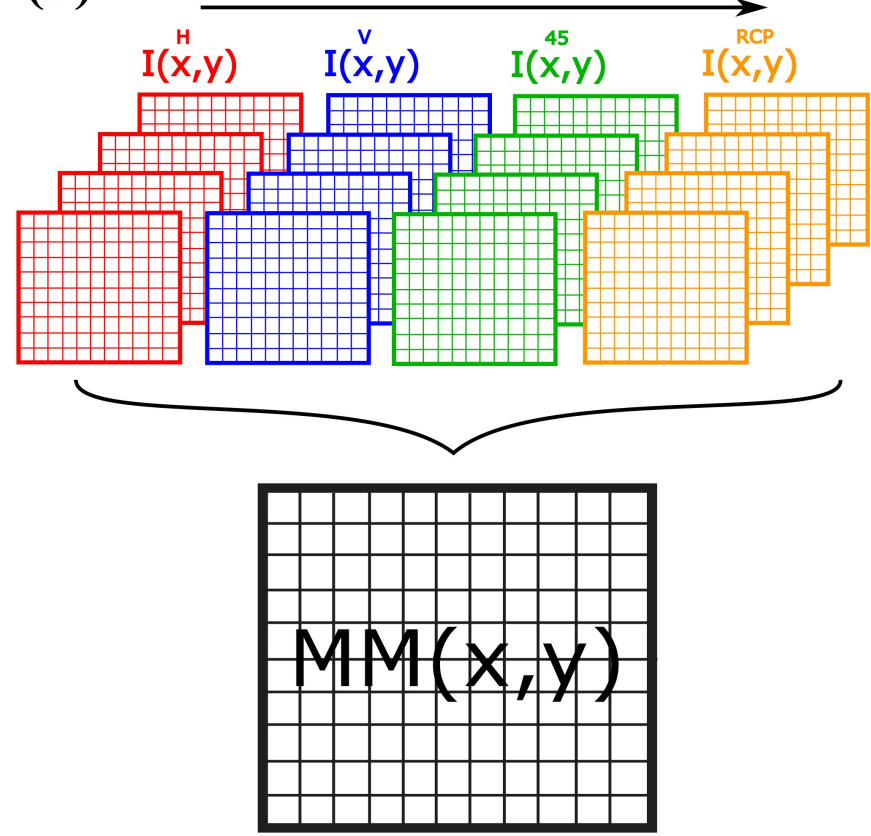

Figure 2. (a) Block diagram of a typical MM SLM configuration based on the sequential encoding/decoding of the polarization states from Reference [45]. (b) Schematic presenting the extraction of the MM images from the measured intensities images in the sequential approach. PSG: polarization states generator. PSA: polarization states analyzer. Obj: microscope objective. Cond: Condenser. GS: galvanometric scanner. TL: tube lens. S: sample. PMT: Photomultiplier tube. t: temporal polarization states generation. $I^{H}, I^{V}, I^{45}$, $I^{R C P}$ : intensities collected at the generated horizontal, vertical, $45^{\circ}$ and Right Circular Polarization (RCP) polarized states.

In the confocal mode, an aperture slightly smaller in diameter than the Airy disc image is positioned in the image plane in front of the detector [46]. The ability of this method to reduce out-of-focus blur signal, and thus permit accurate non-invasive optical sectioning that makes confocal scanning microscopy so well suited for the imaging and three-dimensional tomography of biological specimens. It has several additional advantages over conventional optical microscopy, including the possibility of a significant improvement in lateral resolution and the capability for direct non-invasive serial optical sectioning of intact specimens. In 2005, the first confocal Mueller reflection microscope was developed for the retinal diagnosis [27]. The scheme suggested to increase as much as possible the polarization encoding/decoding speed rate allowing the highest number of measurements for improving optically the SNR. For this reason, the generation of the polarization states is performed thanks to two Pockels Cells (PC) allowing the encoding of the polarization in few MHz. In an effort to speed up the decoding time, the method was coupled with a combination of multiple polarization resolved detectors based on the Division of Amplitude (DoA) method [47]. Thus, the time for getting the full MM is around $50 \mathrm{~ms}$ and reach a lateral optical resolution of $30 \mu \mathrm{m}$. However, the implementation of such a technique into multimodal system is difficult considering the physical space required. Furthermore, using multiple polarization optics leads to errors in aligning the optical axis taken into account from the ECM. Nowadays, the multiple reflection optics used for this method, such as beamsplitters, fresnel rhombs or wollaston prism, could be reduced in very miniature modules of few centimeters designing from metasurfaces [48].

In 2008, an already existing commercial transmission SLM was modified for MM reflection imaging of glaucoma [49]. The system was completely automatized based on the use of Liquid Crystal Variable Retarder (LCVR) and required 72 successive images 
polarization resolved in $4 \mathrm{~s}$ with $20 \mu \mathrm{m}$ lateral resolution. It is worth noting that such a sample does not exhibit all the polarimetric changes in presence of a pathology, decreasing the number of acquisition needed for studying only few polarimetric parameters.

More recently, a MM polarimeter has been implemented into a non-linear commercial transmission scanning microscope using simple motorized optical devices [50]. It allows the acquisition of only four images coded by four distinct polarization states (horizontal, vertical, $45^{\circ}$ and right circular) sequentially also based on DoA method. The main application was the study of starch molecules under certain conditions (thermal and mechanical stress). Similarly, a similar approach has been extended for small animal embryonic development analysis in the reflection configuration [45].

The ability of such a technique paves the way of acquiring the full MM images of the sample simultaneously with non-linear confocal microscopy due to its compactness and versatility. This could be of interest for tracking the source of the label-free contrasts for thick objects where the polarimetric imaging modality gives only an average information of the scattering sample fingerprint through the PSF volume. A promising advance in this direction has been proposed in 2020 for characterizing scattering media in MM reflectance configuration in a confocal mode [51]. The instrument demonstrated is capabilities by measuring the increasing of depolarization through highly scattering medium such as milk solution. The polarimetric changes of layered-like structure has been validated through Monte Carlo simulations and showed that the depolarization and retardance caused by a birefringent fiber is influenced by its shape and location. This work has been completed by performing a depth-resolved imaging of cornea that combines here Mueller matrix in both transmission and reflection configuration with non-linear microscopy utilizing Two-Photon Excitation Fluorescence (TPEF) and Second Harmonic Generation (SHG) [52]. In this work, both the PSG and PSAs are composed of a pair of LCVRs and a linear polarizer and the voltages are synchronized with the GS. Then, the MM images are extracted pixel-by-pixel based on the measurements of four different PSA states for each of the six PSG states, so a set of 24 images is obtained. These promising results show the capability of extracting the polarimetric contrasts at a specific depth through the sample randomly organized. Indeed, they demonstrate that the random changes in the corneal model as a layered medium in the order of micrometer can strongly affect its polarization properties. However, it is worth noting that these preliminary results deal with pure deterministic samples (here only birefringent) where the measured scattering results mainly from the accumulation of random orientation across the illumination volume.

\subsection{Spectral Domain Encoding/Decoding}

The previous techniques are based on acquiring a set of a sequentially generated polarization resolved images and recombined them in a post data process for recovering the full MM image, although this approach is not suitable for any dynamic characterization of the sample limiting the use of such a technique for in vivo microscopy imaging. Indeed, the MM polarimeter should be able of acquiring the full MM in the pixel dwell time by mean in few microseconds and requires a very fast intensity modulation of the polarized light.

For wide field imaging, the solution has been found by using multiple electro-optics devices in series such as Photoelastic Modulators (PEMs) or PC triggered with the camera frame rate via a data acquisition (DAQ) board [53]. Briefly, the method is based on the analysis of the time-variation of the intensity induced by the electro-optics effect. Thus, the detected signal is a channeled spectrum represented in the Fourier domain by complex modulation amplitudes at numerous frequencies. A $50 \mathrm{MHz}$ FPGA counts the edges of each PEM modulation signal and locks when a unique phase between all the four PEMs is occurring within a short time in a nanosecond timescale. Then, the FPGA sends a trigger to the CCD to gate the signal in $0.5 \mu$ s, giving a full image acquisition took in approximately $20 \mathrm{~ms}$. Another approach consisting in dealing directly with the Fourier transform of such modulated signal where the complex amplitudes are linear combinations of the MM elements [54]. In this method, the common approach is to choose different PEMs with 
separated working frequencies. This kind of optical device is composed of a passive crystal subjected to periodic mechanical stress, resulting in a time-varying birefringence due to the photoelastic effect $[28,55]$. In numerous earlier works, polarization-resolved setups used one PEM with an LP oriented orthogonally as a PSG for measuring the differential circular polarized intensities $[33,36,56]$. Coupled with a lock-in detection at the reference frequency of the PEM, the passive setup was able to provide few elements of the MM in order of tens of $\mathrm{kHz}$. In order to speed up the acquisition rate and the number of elements, common solutions proposed to add another PEM in the PSA synchronized with the first one [57]. In recent works, this technique upgraded such a measurement by dealing with 3 and 4 PEMs [58]. The main advantage brought by adding multiple PEMs is that all the elements of the MM can be retrieved without any mechanical moving part. Thus, this experimental approach has been proven to be of interest for studying ultrafast conformational changes in biopolymers [59,60]. For such a technique, the acquisition speed has reached $100 \mu \mathrm{s}$ (10 kHz repetition rate), very close to the pixel dwell time from any GS.

Even if the speed for acquiring the full MM image is fast, a new method for fast polarization encoding has recently emerged and is based on spectral coding of polarization (channeled spectropolarimeters) by using passive elements such as birefringent plates [61]. The principle is the parallelization of polarization states in the spectral domain so that the polarimetric response of a sample can be calculated when using a single-channel spectrum $\mathrm{I}(v)$, where $v$ is the optical frequency. Each channeled spectrum $\mathrm{I}(v)$ is periodic and is composed of discrete frequencies from 0 to 12.f0 that are integer multiples of the fundamental one $\mathrm{f} 0$. This last one depends on both the thickness e and birefringence $\Delta \mathrm{n}$ of the retarders by the relation $\mathrm{f} 0 \Delta \mathrm{ne} / \mathrm{c}$, where c is the celerity of light in vacuum. Such polarimeters have the potential to perform polarimetric scanning microscopy thanks to their speed provided that the thickness ratio of the retarders is well-chosen [62]. The first experimental snapshot Mueller polarimeter based on spectral coding of polarization using a broad spectrum source (superluminescent diode), thick retarder plates, and a CCDbased spectrometer [63]. At this early stage, the device was developed in a non-imaging transmission configuration but recently upgraded for SLM polarization-resolved SHG [64]. The acquisition rate of this approach is limited by only the spectrometer performances that can be high (hundred of MHz). Then, inspired by OCT technology, the technique has been upgraded by using a wavelength-swept laser source, high order retarders, and a single-channel detector [65]. The device uses a wavelength-swept source (SS) laser instead of the broad spectrum source and a photodiode instead of the spectrometer, which results in a much simpler optical setup as shown in Figure 3a. For instance, the PSG and the PSA composed of simple polarization features (linear polarizers and retarder slices) have been compacted in mechanical blocks with centimeter dimensions [31,66]. A singlepoint Mueller matrix is measured at the rate of the SS, which could be hundreds of $\mathrm{kHz}$. This compactness allows for straightforward implementation on a commercial scanning microscope and has been upgraded in transmission into a commercial SLM already use for performing TPEF and SHG imaging [31].

In this work experiment, the fundamental thickness e was chosen to generate six modulations at the frequency f0. This rather large spectral analysis window could be reduced by using thicker retarders but at the expense of the number of samples per channeled spectrum. The channeled intensity spectrum $I(v, t)$ is modulated by the polarimetric fingerprint of the sample and can be expressed as

$$
I(v, t)=\sum_{k=0}^{n}\left[a_{k, t} \cdot \cos \left(k \frac{2 \pi \Delta n e}{c} v\right)+b_{k, t} \cdot \sin \left(k \frac{2 \pi \Delta n e}{c} v\right)\right]
$$

where $a_{k, t}$ and $b_{k, t}$ are linear combinations of Mueller matrix elements $m_{i j}$. In the configuration resumed Figure $3 \mathrm{~b}$, the sum stops at $\mathrm{n}=12$ leading to 12 Fourier amplitudes in real and imaginary part, giving finally 25 values used to retrieved the 16 Mueller elements simultaneously. The same philosophy has been proposed also in a multimodal approach for label-free cancer cervix study [67] by coupling MM wide field imaging with optical 
scanning OCT. Despite the extraordinary advantage offers by the speed of this technique, an important assumption is that the sample is achromatic within the excitation wavelength range. Additionally, the modeling of the instruments assumed that all the linear retarders used do not present any diattanuation or depolarization. Thus, a precise validation of the polarimetric properties of the sample should be done in the working spectral range. Finally, thanks to the high speed rate and the spectral information that give access to numerous measurable quantities at the same time, this method has been successfully proposed for real-time imaging through an endoscopic approach [66]. The main issue of endoscopic technique polarization-resolved is decoupling at any time the signatures of the sample from the optical fiber polarization transformation.
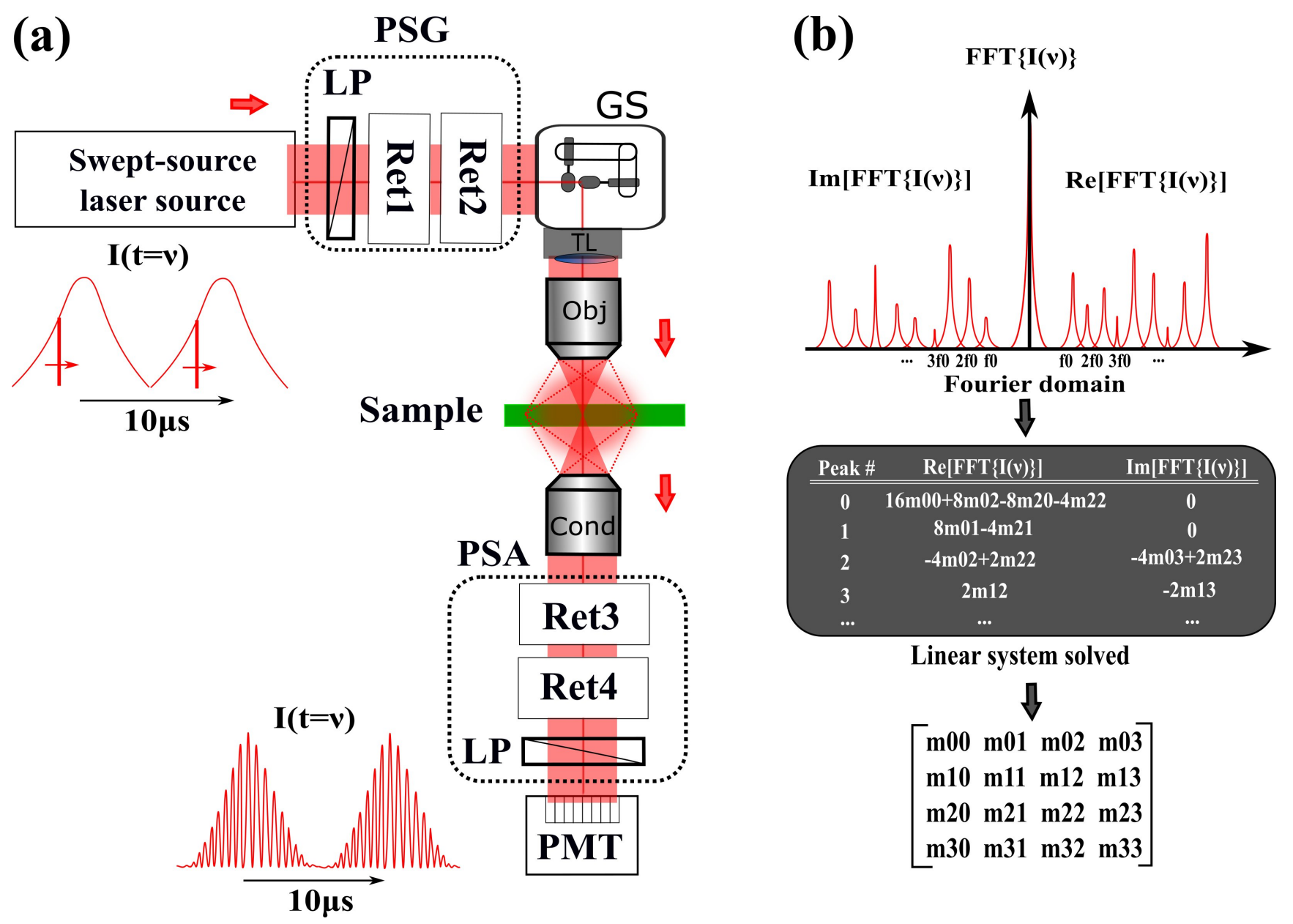

Figure 3. (a) Block diagram of a MM SLM based on the spectral encoding/decoding of the polarization states from Reference [31]. (b) Schematic presenting the extraction of the MM images of the measured intensities images in the spectral approach. PSG: polarization states generator. PSA: polarization states analyzer. Reti: retarder. Obj: microscope objective. GS: galvanometric scanner. TL: tube lens. S: sample. PMT: photomultiplier tube.

We have summarized the typical performances reached by the methods presented in this section in Table 1. Additionally, we have reported the acquisition speed for the $16 \mathrm{MM}$ images in Figure 4. 
Table 1. Main performances and capabilities of the single and multiple channel temporal MM acquisition (two first lines) comparing to the spectral detection for recovering the full Mueller matrix image. The number of scan reported in the first column referred to the minimal number for retrieving the the 16 elements of the MM image. The speed referred to the lowest one for the method reported in the literature.

\begin{tabular}{|c|c|c|c|c|}
\hline & Num. of Scan & Speed & Advantages & Disadvantages \\
\hline Single detection & 16 & few mins & $\begin{array}{c}\text { Simple calibration } \\
\text { Cheap }\end{array}$ & $\begin{array}{l}\text { Precise alignment } \\
\text { Active elements } \\
\text { Succesive scans } \\
\text { Post processing }\end{array}$ \\
\hline Multiple detection & 4 to 8 & $50 \mathrm{~ms}$ to $10 \mathrm{~s}$ & $\begin{array}{c}\text { Snapshot detection } \\
\text { Cheap }\end{array}$ & $\begin{array}{l}\text { Precise alignment } \\
\text { Succesive scans } \\
\text { Heavy calibration } \\
\text { Spacy }\end{array}$ \\
\hline Spectral detection & 1 & $10 \mu \mathrm{s}$ & $\begin{array}{c}\text { Snapshot } \\
\text { Compact } \\
\text { Passive elements }\end{array}$ & $\begin{array}{l}\text { Precise alignment } \\
\text { Heavy calibration } \\
\text { Complex modeling }\end{array}$ \\
\hline
\end{tabular}

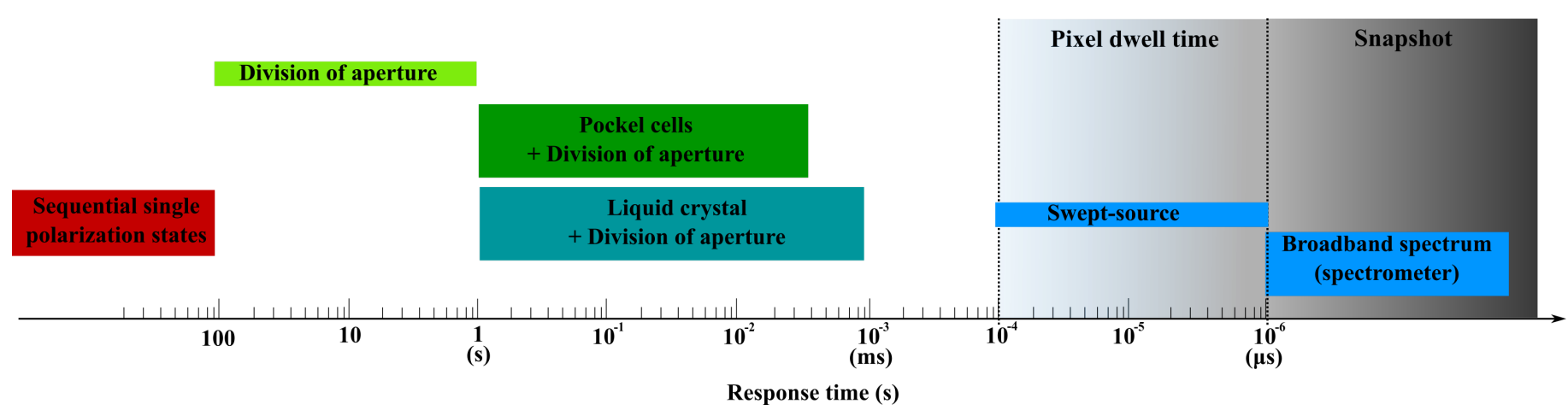

Figure 4. Comparative acquisition speed for measuring the full MM images using the sequential/spectral approaches according to the optical SLM architecture presented in the literature.

\section{Mueller Matrix Applications}

MM microscopy has proven to be a promising tool for a wide range of applications, mostly used for biomedical diagnosis. It is explained by the need of imaging at a high speed rate any tissue modification induced by the presence of a pathology in a non-invasive way without any labelling process. Additionally, this research field required to investigate at distance and from outside in vivo pathological signature in fast way. This has been of interest for characterising micro-patterned structures and layered metasurfaces, that requires a fast and an automatized process. For utilizing this technique from the biomedical diagnosis into industrial applications, the MM imaging experimental architecture is almost the same. The main difference comes from the interpretation of the polarimetric response of the samples. Indeed, it is well stated that biological media exhibits stronger depolarization than solid-state structures due to the random organization overall the illumination volume [11]. This leads to an arduous task in tracking the source of the contrast at a specific depth, limiting this technique nowadays for the direct use in clinical applications, such as for the histopathology.

\subsection{Ophthalmology}

As presented in the last section, the main application of MM microscopy using SLM was devoted to ophthalmology. The main reason comes from the desire in finding an alternative approach for diagnosing any pathology at rate faster than the eye motion similar to earlier methods proposed by OCT imaging. Moreover, coupling with additional signal processing, MM offers the reduction of the exposure time that limit the damage through the 
eye. Besides, it has been shown that in presence of pathology, the disorganization of thick tissues exhibits strong modification in the birefringence and depolarization and MM has the capability of quantifying these parameters. This explains that most of the incomplete MM microscopes tends to track these only two parameters reducing the number of information but accelerating the process for encoding/decoding the polarization states $[68,69]$.

First, because the ocular media and the retina in the human eye exhibit rather complicated polarization properties, every technique based on collecting the light scattered back into the retina in double pass is affected by polarization. Second, another challenge for such a system is the fast motion and optical properties in the living eye, required a high speed rate encoding/decoding of the polarization states. Third, to avoid any damage, the wavelength should be chosen wisely so as to reduce the radiation exposition and limit the absorption that participates in reducing drastically the polarimetric SNR. Some images obtained in the SLM configuration for MM are presented Figure 5.

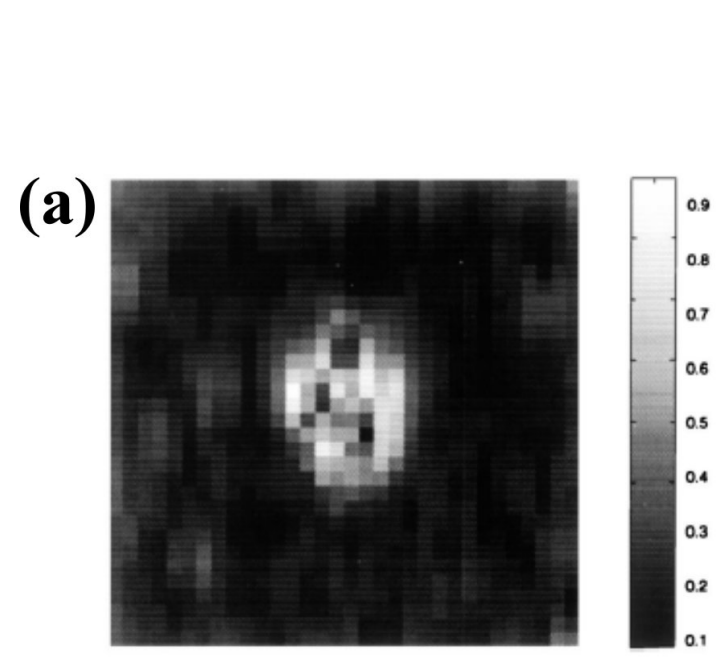


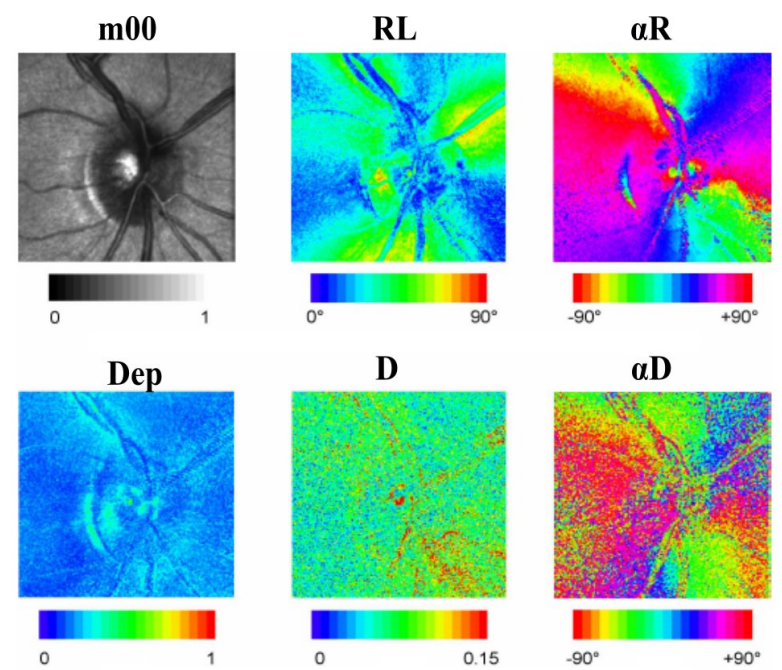

$\alpha \mathbf{D}$
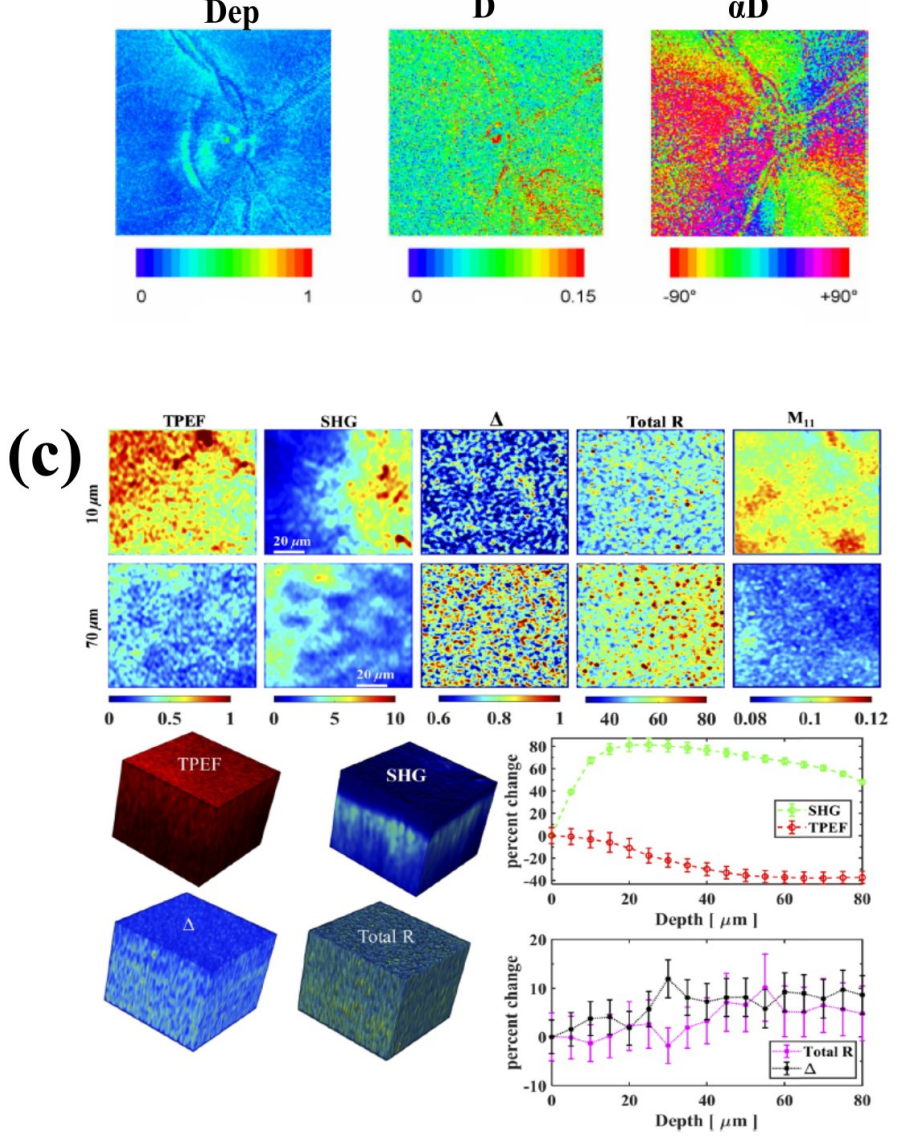

Figure 5. Quantitative imaging of the full Mueller matrix for ophtalmology applications. (a) Degree of polarization images of the retina for two different size of pupil. (b) Polarization parameter images of the optical nerve head. These images corresponds to the normalized average intensity m00; linear retardance- $\mathrm{RL}$; retardance orientation $\alpha_{R}$; depolarization index Dep; diattenuation D; diattenuation orientation $\alpha_{D}$. and (c) Quantitative analysis of a rat cornea polarization resolved image. Top two rows correspond to images at 10 and $70 \mu \mathrm{m}$ depth while bottom row shows 3D volume of Second Harmonic Generation (SHG) and Two-Photon Excitation Fluorescence (TPEF), and percent change. Adapted with permission from: (a) [44]; (b) [49]; (c) [52], (C) The Optical Society. 
One of the first application of MM has been proposed in 2000 that studied the influence of the eye pupil size on the birefringence [70]. For limiting the exposure time but acquiring a high number of image, the number of pixel is quite small. Furthermore for such an application, the numerical aperture could not be high for investigating at distance the medium limiting drastically the optical resolution. The quantification of the polarimetric properties of the different part of the eye (optic nerve, macula, cornea and retina) is shown in Figure 5b. As it can be noted in this application, the contrast distribution for some parameters are completed blurred by the coupling effect of the back reflection and highly scattering regions in the eye. This is challenging where tracking slight changes at early pathological stages in a low SNR area is mandatory. Recent works in 2020 shown in Figure $5 \mathrm{c}$ have successfully overcome this issue by acquiring the elementary MMs in $80 \mu \mathrm{m}$ depth using a confocal approach [52]. This study has been performed on extracted rat cornea in both transmission and reflection configuration. The main purpose was to investigate the propagation of the polarimetric changes through a highly scattering environment layer-by-layer induced by the random distribution of collagen and cells. Additionally, with the purpose of tracking the source of the polarimetric contrasts, MM imaging acquisition is coupled with non-linear imaging modalities and has been coupled with Monte Carlo simulations. As expected, the study reveals that the depolarization and retardance increase in depth and that a high polarization heterogeneity can be noted in $\mathrm{xy}$ and $\mathrm{z}$ direction since the data collected correspond to average values through the confocal volume. However, this heterogeneity in the polarization properties are not only explained by the random distribution of the localized biological components, but also by the influence of measured metrics, such as the glucose level in the blood. This results in extremely challenging issue for decoupling the optical rotation from glucose from the pure deterministic polarimetric response of the layered biological arrangement.

\subsection{Biomedical Diagnosis and Tissue Organization}

Recently, many works have been focused on developing MM polarimeter to investigate the modification of the tissues induced by pathology at the cellular level [71-73]. Indeed, MM offers the capability of tracking changes at distance in a non-invasive and label free way since no high power is required for detecting the polarized light. Coupling with SLM, a better contrast emerged from this technique, resolving sub-microscopic objects optical properties giving the capability of quantifying pathologies at early stages.

Cancers induce drastic modifications in cells size and collagen organization. Actually, the scoring of pathologies are performed by specialists and the accuracy of the analysis in completely operator dependant. This is the reason that the recent emerging methods proposed to automatize the measurements and the interpretation of the results through statistical interpretation and machine learning. The approach is almost the same and consists in measuring the associated physical changes to the cancer that exhibit strong polarimetric effects comparatively to the healthy area. Thus, MM provides an interesting method for imaging confined localized structures and bring quantitative methods for staging the pathologies. It is the purpose of the applications proposed in Figure 6.

More particularly, the work in Figure 6i proposed a statistical approach for scoring liver fibrosis biopsis by analyzing the retardance and depolarization parameters compared to the SHG response. This pathology induces an accumulation of the type I and III fibrillar collagen through the extracellular matrix in the hepatic tissue. The non-linear signal erases from the anisotropic arrangement in the collagen structure and are correlated to the retardance images as observed. Based only on this parameter, it is impossible to distinguish the different arrangement in the collagen fibers, different from the random accumulation in the fibrosis or from the organized collagen composing blood vessels. Based on a pure visual interpretation, this can leads a bad scoring of the fibrosis. However, it is well stated that the random distribution of deterministic objects (here pure birefringent) leads to depolarization, MM gives the opportunity of discriminating the two kinds of organization through this parameter. Furthermore, Figure 6ii shows that this discrimination can be done 
on the image extracted from the retardance orientation (i.e., birefringent fast axis) where it is possible to follow the rotation of the fiber in space with high imaging contrast. It could be of interest in the case the experiment requires the tracking of a specific polarimetric change under certain conditions as it has be done for fixed zebrafish reported in Figure 6iii [45]. This work has proposed to acquire a detailed fingerprint at different developmental stages and interpret all the MM parameters. This is key in understanding the morphological changes of the animal during development. It has been shown that each parameters could be use as a specific markers for local structures since the physical effects are reliable to a dedicated structure and organization. For zebrafish, it has been proven than the imaging contrast from $\mathrm{Pd}$ comes from the thickness heterogeneity while the $\mathrm{R}$ values erases from the tissues and muscles.

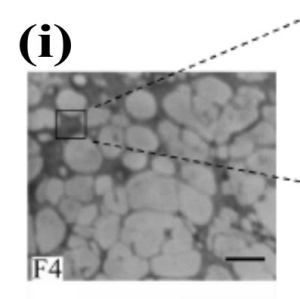

NP image

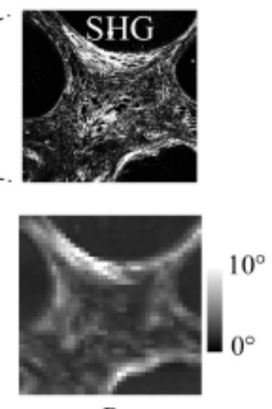

$\mathrm{R}$

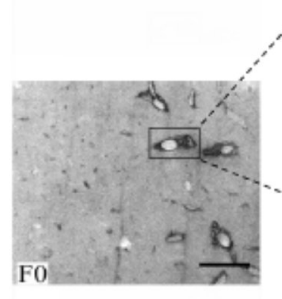

NP image

(ii)

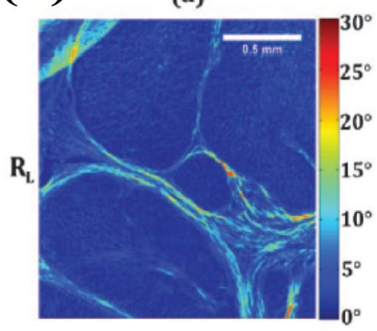

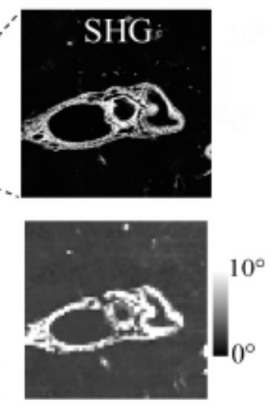

$\mathrm{R}$ (iii)

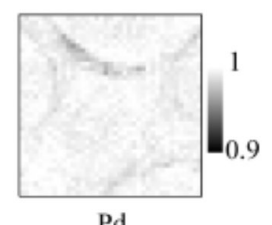

Pd

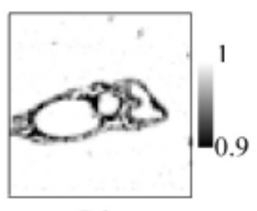

Pd

(b)

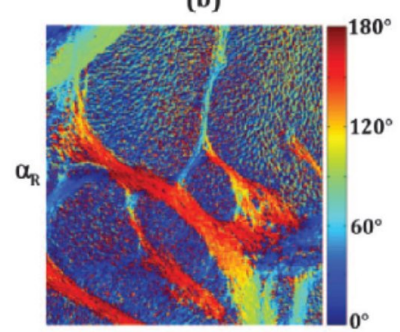

(a)
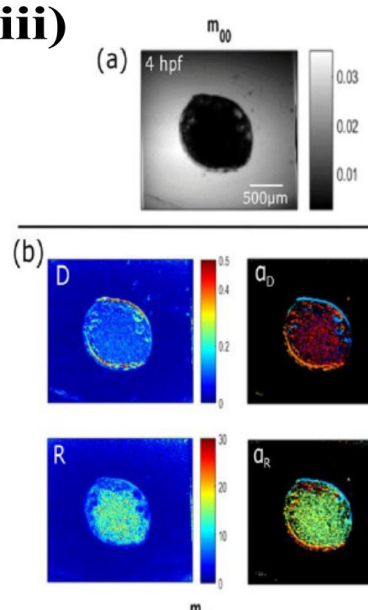

(e)
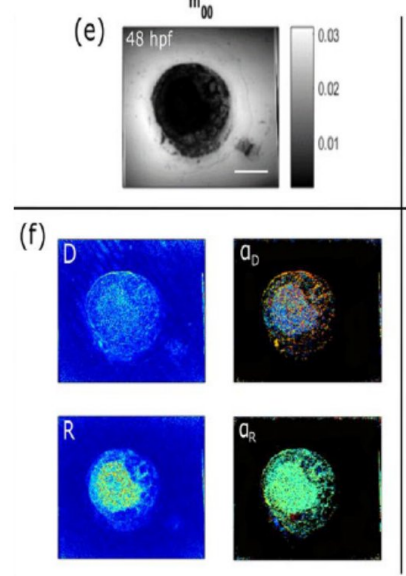

(c)



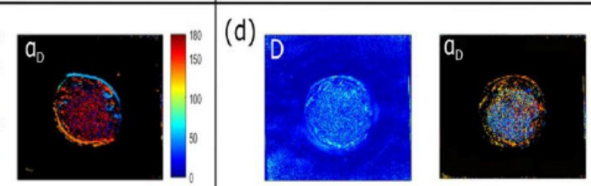
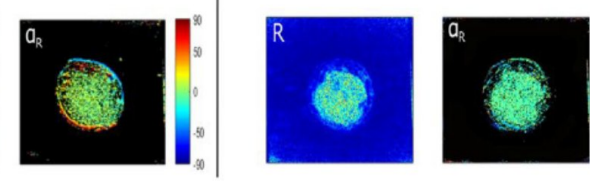

(g)
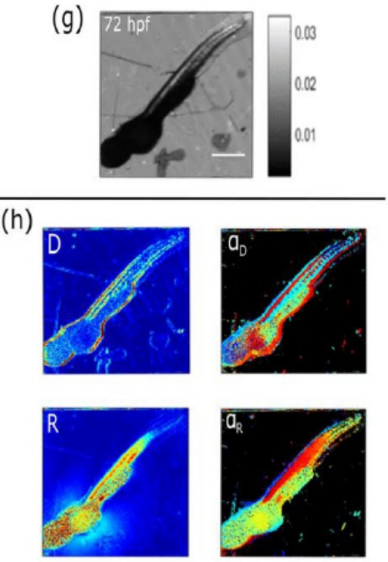

Figure 6. Quantitative imaging of the full MM images for biomedical diagnosis. (i) Non-Polarized (NP), SHG, Retardance (R) and depolarization index (Pd) images of stained surgical human liver biopsies (F0 and F4 Metavir grades). (ii) Retardance (R) and its orientation $\left(\alpha_{R}\right)$ image of a human liver biopsy. (iii) Polarimetric images of fixed zebrafish embryos and larvae at 4 hpf (hours post fertilization), $24 \mathrm{hpf}, 48 \mathrm{hpf}$ and $72 \mathrm{hpf}$. The images (a), (c), (e), (g) correspond to the total collected intensity from the MM, which is the m00 element. The images (b), (d), (f), (h) are images coded in Diattenuation (D), in Retardance and its azimuthal orientations $\alpha_{D}$ and $\alpha_{R}$, respectively. Adapted with permission from: (i) [74]; (ii) [31], (C) The Optical Society; (iii) [45], Scientific Reports, under a Creative Commons license (http:/ / creativecommons.org/licenses/by/4.0).

\subsection{Material Science}

Besides these main applications for the biomedical diagnosis, the characterization of the full optical property of nanostructures at high speed rate and in a cheap way offered 
by SLM is suitable in real-world industrial manufacturing applications. The main challenge of this application is the capability of characterizing the sample at the nanoscale. In polarimetry, most of the ellipsometers are dedicated for characterizing thin films in microelectronics [75-77]. However, this approach is based on the analysis of the diffraction pattern in widefield imaging and are compared with models using elementary decomposition of the MM $[78,79]$.

The characterization of nanostructures has been widely studied by electron microscopy or in a non-linear optical approach. These approaches provides valuable information related to the sample conformation at the nanoscale but are based on the high power light dose absorption and are invasive in term of sample preparation. MM has proven to overcome these issues and its easy experimental implementation gives the opportunity of tracking live polarimetric changes. One of the few examples in the literature related to in vivo live imaging using SLM has been proposed in Reference [50] as shown in Figure 7a.

(a)
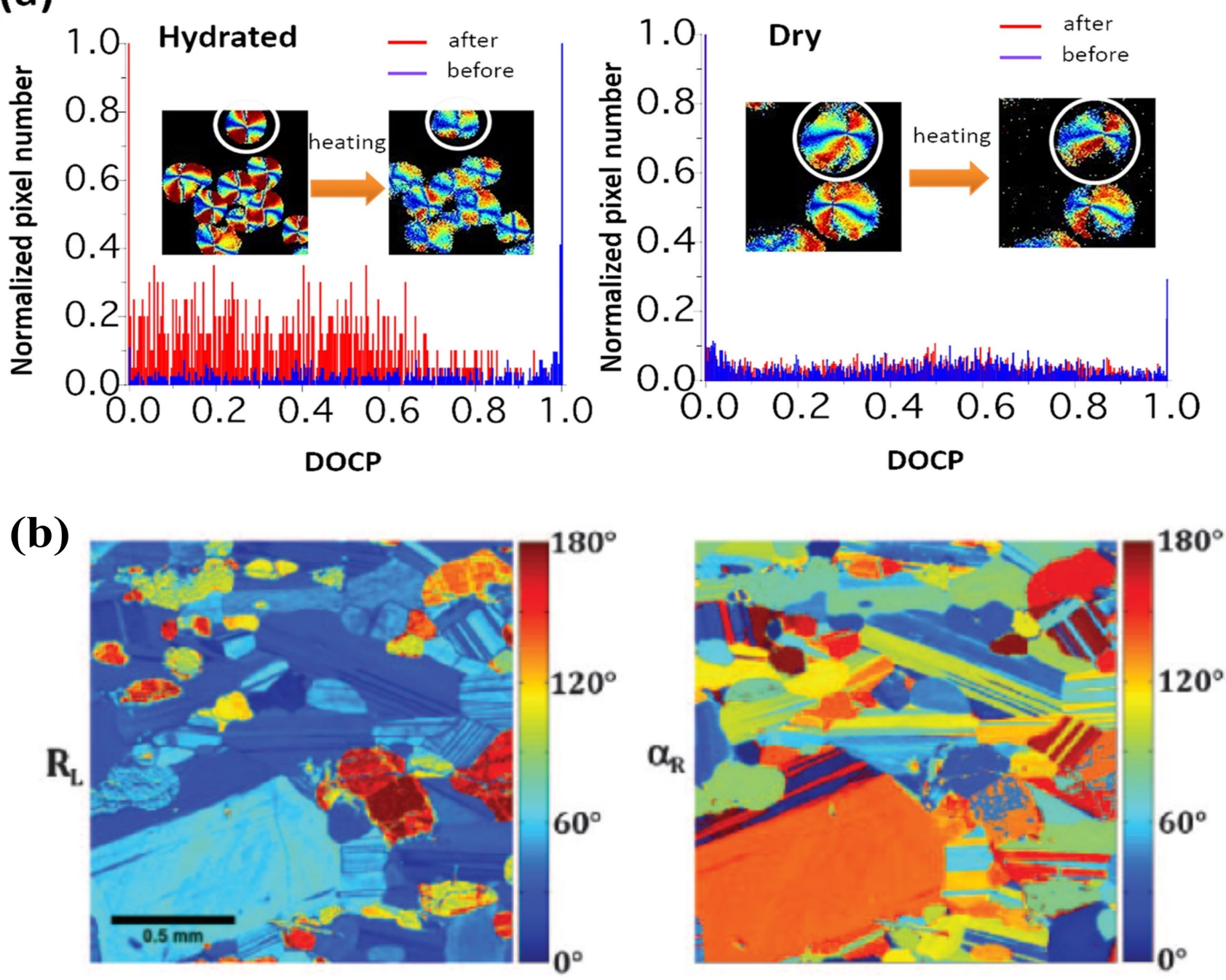

Figure 7. Quantitative MM images taken from material science applications. (a) Comparative study of the SHG images of hydrated and dry starch granules after heating. (b) Linear retardance RL, and its orientation $\alpha_{R}$ of a gabbro rock section. Adapted with permission from: (a) [50], Scientific Reports, under a Creative Commons license (http:/ / creativecommons.org/licenses/by/4.0); (b) [31], (C) The Optical Society.

This work consisted in studying the polarimetric modifications of starch granule under certain conditions (thermal, hydration level and temporal). Indeed, starch is of interest due 
to its easy, cheap availability and its concentric shell structure forms a natural photonic crystal structure easy to model. In the past year, studying the starch structures has been of interest since it is a major source of our daily diet and its conformation plays a significant role for the quality of food. In this work, the denaturation and the phase transition after a thermal change has been studied through the Degree of Polarization (DOP) Linear (DOLP or Circular (DOCP) indicating that starch has a greater degree of ultrastructural amylopectin order. It has proven that the degradation at the intramolecular level occurs in hydrated starch whereas disruption of surface in dry only while no significant change of polarization properties are observed at higher temperatures in case of dry starch. Thanks to the wide range of parameters provided by $\mathrm{MM}$, other physical quantities could be use for interpreting crystal at the microscopic level as shown in Figure $7 \mathrm{~b}$. Conventional polarization microscopy study the polycrystalline structures of rocks using simple cross polarizers. It reveals the presence of different minerals by rotating the sample, resulting in intensity changes which comes from the difference in birefringence and the thickness of the crystals. The advantage of moving into an MM configuration is the capability of quantifying the absolute value of $\mathrm{D}$ and as well as the orientation of the mineral without moving parts.

\section{Conclusions}

Mueller matrix microscopy is an interesting label-free approach for understanding the organization of any medium that demonstrate its potentiality in the many fields. This technique has multiple advantages over other traditional optical imaging modalities, such as being a versatile and flexible tool, with low complexity and low cost. More particularly, we have presented some methods using miniaturized and passive encoding/decoding modules of the polarization, easier to placed inside any microscope body. Thus, the use of low power laser source and cheap photodetectors prove that this technique could be an affordable tool by any research group. In this review, we presented the earliest and the most advanced numerical models that have proven their accordance to experimental data. We showed that the architectures are inspired by Mueller-Stokes setups simple to design and build. However, at such scale, it could be arduous to identify properly the source of the contrast since the polarimetric changes occur at every elementary layers in the illumination volume. Additionally, switching from the single point configuration into an imaging system is not straightforward, and different sources of optical artifacts could pollute the measurements. These come from the polarization transformation through the whole optical setup that alter the polarization quality and hide the true sample fingerprint. It becomes even more challenging when all these sources of error have to be evaluated at rate fast enough to be suitable with the scanning beam approach. Thanks to the recent advances and demonstrations offered by the upgrade of the SLM, this approach shows its capability to be easily implemented in different optical architecture, providing simultaneous diverse source of contrasts. Furthermore, it is easy to imagine 3D real-time measurement in the polarization-based imaging research field.

Funding: This research received no external funding.

Acknowledgments: The authors thank the Nikon Imaging Center at the Fondazione Istituto Italiano di Tecnologia (Genoa, Italy) for the support in the realization of the experiment. We thank Colin Sheppard for the fruitful discussions about the Mueller matrix formalism.

Conflicts of Interest: The authors declare no conflict of interest. 




\section{References}

1. McMaster, W.H. Polarization and the Stokes Parameters. Am. J. Phys. 1954, 22, 351-362. [CrossRef]

2. Huard, S. Polarization of Light. In Polarization of Light, 1st ed.; John Wiley \& Sons Inc.: New York, NY, USA, 1997.

3. Anche, R.; Sen, A.K.; Anupama, G.; Sankarasubramanian, K.; Skidmored, W. Analysis of polarization introduced due to the telescope optics of the Thirty Meter Telescope. J. Astron. Telesc. Instrum. Syst. 2018, 4, 01800. [CrossRef]

4. Patty, C.; ten Kate, I.; Jan Buma, W.; van Spanning, R.; Steinbach, G.; Ariese, F.; Snik, F. Circular spectropolarimetric sensing of vegetation in the field: possibilities for the remote detection of extraterrestrial life. Astrobiology 2019, 19, 1221-1229. [CrossRef]

5. Tyo, J.; Goldstein, D.; Chenault, D.; Shaw, J. Review of passive imaging polarimetry for remote sensing applications. Appl. Opt. 2006, 45, 5453-5469. [CrossRef]

6. Zhai, P.; Hu, X.; Trepte, C.; Lucker, P. A vector radiative transfer model for coupled atmosphere and ocean systems based on successive order of scattering method. Opt. Express 2009, 17, 2057-2079. [CrossRef]

7. Fade, J.; Panigrahi, S.; Carre, A.; Frein, L.; Hamel, C.; Bretenaker, F.; Ramachandran, H.; Alouini, M. Long-range polarimetric imaging through fog. Appl. Opt. 2014, 53, 3854-3865. [CrossRef] [PubMed]

8. Goudail, F.; Boffety, M.; Roussel, S.; Lucker, P. Optimal configuration of static Mueller imagers for target detection. J. Opt. Soc. Am. A 2017, 34, 1054-1062. [CrossRef] [PubMed]

9. Hielscher, H.; Eick, A.; Mourant, J.; Shen, D.; Freyer, J.; Bigio, I. Diffuse backscattering Mueller matrices of highly scattering media. Opt. Express 1997, 1, 441-453. [CrossRef] [PubMed]

10. Sankaran, V.; Walsh, J.; Maitland, D. Comparative study of polarized light propagation in biologic tissues. J. Biomed. Opt. 2002, 7. [CrossRef] [PubMed]

11. Tuchin, V.; Wang, L.; Zinnyakov, D. Polarized light interaction with strongly scattering media. Opt. Polariz. Biomed. Appl. 2006, 4, 45-67.

12. Anna, G.; Goudail, F.; Daniel, D. Polarimetric target detection in the presence of spatially fluctuating Mueller matrices. Opt. Lett. 2011, 36, 4590-4592. [CrossRef]

13. Doronin, A.; Macdonald, C.; Meglinski, I. Propagation of coherent polarized light in turbid highly scattering medium. J. Biomed. Opt. 2014, 19, 025005. [CrossRef] 
14. Wang, H.; Hu, H.; Xiaobo, L.; Guan, Z.; Zhu, W.; Jiang, J.; Liu, K.; Liu, T. An Angle of Polarization (AoP) Visualization Method for DoFP Polarization Image Sensors Based on Three Dimensional HSI Color Space. Sensors 2019, 19, 26. [CrossRef]

15. Mueller, H. The foundations of optics. J. Opt. Soc. Am. 1948, 38, 661-662.

16. Snik, F.; Craven-Jones, J.; Escuti, M.; Fineschi, S.; Harrington, D.; De Martino, A.; Mawet, M.; Riedi, J.; Tyo, J. Measurement of the optical activity of anisotropic samples by transmission Mueller matrix ellipsometry. Polariz. Meas. Anal. Remote Sens. XI 2014, 9099, 9099B.

17. Qi, J.; He, C.; Elson, D. Real time complete Stokes polarimetric imager based on a linear polarizer array camera for tissue polarimetric imaging. Biomed. Opt. Express 2017, 8, 4933-4946. [CrossRef] [PubMed]

18. Lapray, P.; Gendre, L.; Foulonneau, A.; Bigué, L. An FPGA-based pipeline for micropolarizer array imaging. Int. J. Circuit Theory Appl. 2018, 46, 1675-1689. [CrossRef]

19. Rankin, D.; Matthies, L.; Hertas, A. Daytime Water Detection by Fusing Multiple Cues for Autonomous Off-Road Navigation. In Proceedings of the 24th Army Science Conference, Orlando, FL, USA, 29 November-2 December 2004.

20. Hasegawa, R.; Matsusaka, S.; Hidai, H.; Chiba, A.; Morita, N.; Takashi, O. In-process estimation of fracture surface morphology during wheel scribing of a glass sheet by high-speed photoelastic observation. Precis. Eng. 2017, 48, 164-171. [CrossRef]

21. Rebhan, D.; Rosenberg, M.; Notni, G. Principle investigations on polarization image sensors. Photonics Educ. Meas. Sci. 2019. [CrossRef]

22. Powell, S.; Gruev, V. Calibration methods for division-of-focal-plane polarimeters. Opt. Express 2013, 21, 21039-21055. [CrossRef] [PubMed]

23. Chen, C.; Chen, X.; Shi, Y.; Gu, H.; Jiang, H.; Liu, S. Metrology of Nanostructures by Tomographic Mueller-Matrix Scatterometry. Appl. Sci. 2018, 8, 2583. [CrossRef]

24. Stokes, G. On the composition and resolution of streams of polarized light from different sources. Trans. Camb. Philos. Soc. 1992, 9, 339-416.

25. Compain, E.; Poirier, S.; Drevillon, B. General and self-consistent method for the calibration of polarization modulators, polarimeters, and Mueller-matrix ellipsometers. Appl. Opt. 1999, 38, 3490-3502. [CrossRef]

26. Laude-Boulesteix, B.; De Martino, A.; Drevillon, B.; Schwartz, L. Mueller polarimetric imaging system with liquid crystals. Appl. Opt. 2004, 43, 2824-2832. [CrossRef]

27. Lara, D.; Dainty, C. Axially resolved complete Mueller matrix confocal microscopy. Appl. Opt. 2006, 45, 1917-1930. [CrossRef] [PubMed]

28. Kemp, J. Piezo-optical birefringence modulators: new use for a long-know effect. J. Opt. Soc. Am. 1969, 59, 950-954. [CrossRef]

29. Sabatke, D.; Descour, M.; Dereniak, E.; Sweatt, W.; Kemme, S.; Phipps, G. Optimization of retardance for a complete Stokes polarimeter. Opt. Lett. 2000, 25, 802-804. [CrossRef] [PubMed]

30. Macias-Romero, C.; Torok, P. Eigenvalue calibration methods for polarimetry. J. Eur. Opt. Soc. Rapid Publ. 2012, 7, 12004. [CrossRef]

31. Le Gratiet, A.; Dubreuil, M.; Rivet, S.; Le Grand, Y. Scanning Mueller polarimetric microscopy. Opt. Lett. 2016, 41, 4336-4339. [CrossRef]

32. Schneider, A.; Schneider, M.J.; Rosenheck. Optical Activity of Biological Membranes: Scattering Effects and Protein Conformation. Proc. Natl. Acad. Sci. USA 1970, 66, 793-798. [CrossRef]

33. Dorman, B.; Maestre, M. Experimental differential light-scattering correction to the circular dichroism of bacteriophage T2. Proc. Natl. Acad. Sci. USA 1973, 70, 255-259. [CrossRef]

34. Tinoco, I.J.; Maestre, M.F.; Bustamante, C.; Keller, D. Use of circularly polarized light to study biological macromolecules. Pure Appl. Chem. 1984, 56, 1423-1428. [CrossRef]

35. Wells, K.; Beach, D.; Keller, D.; Bustamante, C. An analysis of circular intensity differential scattering measurements: Studies on the sperm cell of Eledone cirrhosa. Biopolymers 1986, 25, 2043-2064. [CrossRef] [PubMed]

36. Diaspro, A.; Bertolotto, M.; Vergani, L.; Nicolini, C. Polarized light scattering of nucleosomes and polynucleosomes-in situ and in vitro studies. IEEE Trans. Biomed. Eng. 1991, 38, 670-678. [CrossRef] [PubMed]

37. Le Gratiet, A.; Marongiu, R.; Diaspro, R. Circular Intensity Differential Scattering for Label-Free Chromatin Characterization: A Review for Optical Microscopy. Polymers 2020, 12, 2428. [CrossRef] [PubMed]

38. Mickols, W.; Maestre, M.; Tinoco, I.; Embury, S. Visualization of oriented hemoglobin S in individual erythrocytesby differential extinction of polarized light. Proc. Natl. Acad. Sci. USA 1985, 82, 6527-6531. [CrossRef] [PubMed]

39. Keller, D.; Bustamante, C.; Maestre, M.; Tinoco, I. Imaging of optically active biological structures by use of circularly polarized light. Proc. Natl. Acad. Sci. USA 1985, 82, 401-405. [CrossRef]

40. Beach, D.; Wells, K.; Husher, F.; Bustamante, C. Differential polarization microscope using an image dissector camera and phase-lock detection. Rev. Sci. Instrum. 1987, 58, 1987-1995. [CrossRef]

41. Kim, M.; Keller, C.; Bustamante, C. Differential Polarization Imaging I: Theory and Applications. Polariz. Spectrosc. Ordered Syst. 1987, 242, 313-356.

42. Finzi, L.; Ulibarri, L.; Bustamante, C. Differential polarization imaging. V. Numerical aperture effects and the contribution of preferential scattering and absorption to the circular dichroism images. Biophys. J. 1987, 59, 1183-1193. [CrossRef]

43. Gupta, V.; Kornfield, J. Polarization modulation laser scanning microscopy: A powerful tool to image molecular orientation and order. Rev. Sci. Instrum. 1994, 65, 2823-2828. [CrossRef] 
44. Bueno, J.M.; Artal, P. Double-pass imaging polarimetry in the human eye. Opt. Lett. 1999, 24, 64-66. [CrossRef]

45. Le Gratiet, A.; D'Amora, M.; Duocastella, M.; Marongiu, R.; Bendandi, A.; Giordani, S.; Bianchini, P.; Diaspro, A. Zebrafish structural development in Mueller-matrix scanning microscopy. Sci. Rep. 2019, 9, 1-10.

46. Sheppard, C.; Shotton, D. Confocal Laser Scanning Microscopy. In Confocal Laser Scanning Microscopy, 1st ed.; Garland Science: New York, NY, USA, 1997.

47. Azzam, R.M.A. Arrangement of four photodetectors for measuring the state of polarization of light. Opt. Lett. 2008, 10, 309-311. [CrossRef] [PubMed]

48. Intaravanne, Y.; Chen, X. Recent advances in optical metasurfaces for polarization detection and engineered polarization profiles. Nanophotonics 2020, 9, 1003-1014. [CrossRef]

49. Twietmeyer, K.; Chipman, R.; Elsner, A.; Zhao, Y.; VanNasdale, D. Mueller matrix retinal imager with optimized polarization conditions. Opt. Express 2008, 16, 21339-21354. [CrossRef] [PubMed]

50. Mazumder, N.; Xiang, L.; Qiu, J.; Kao, F. Investigating starch gelatinization through Stokes vector resolved second harmonic generation microscopy. Sci. Rep. 2017, 7, 1.

51. Saytashev, I.; Saha, S.; Chue-Sang, J.; Lopez, P.; Laughrey, M.; Ramella-Roman, J. Self validating Mueller matrix Micro - Mesoscope (SAMMM) for the characterization of biological media. Opt. Lett. 2020, 45, 2168-2171. [CrossRef]

52. Le, V.; Saytashev, I.; Saha, S.; Lopez, P.; Laughrey, M.; Ramella-Roman, J. Depth-resolved Mueller matrix polarimetry microscopy of the rat cornea. Biomed. Opt. Express 2020, 11, 5982-5994.

53. Alali, S.; Gribble, A.; Vitkin, I.A. Rapid wide-field Mueller matrix polarimetry imaging based on four photoelastic modulators with no moving parts. Opt. Lett. 2016, 41, 1038-1041. [CrossRef]

54. Alenin, A.; Tyo, J. Generalized channeled polarimetry. J. Opt. Soc. Am. A 2014, 31, 1013-1022. [CrossRef]

55. Hunt, A.; Huffman, D. A new polarization-modulated light scattering instrument. Rev. Sci. Instrum. 1973, 44, 1753-1762. [CrossRef]

56. Bronk, B.; Druger, S.; Czégé, J.; Van de Merwe, W. Measuring diameters of Rod-Shaped bacteria in vivo with polarized light scattering. Biophys. J. 1995, 69, 1170-1177. [CrossRef]

57. Jellison, G.; Modine, F.; Chen, C. Calibration procedures for ellipsometer a two-modulator generalized. Polariz. Meas. Anal. Remote Sens. II 1999, 956, 3754.

58. Arteaga, O.; Freudenthal, J.; Wang, B.; Nichols, S.; Kahr, B. Circular dichroism with multiple photoelastic modulators. Chim. Oggi 2012, 30, 6-9.

59. Narushima, T.; Okamoto, H. Circular Dichroism Microscopy Free from Commingling Linear Dichroism via Discretely Modulated Circular Polarization. Sci. Rep. 2016, 6, 1-10. [CrossRef] [PubMed]

60. Arteaga, O.; El-Hachemi, Z.; Ossikovski, R. Snapshot circular dichroism measurements. Opt. Express 2019, 27, 6746-6756. [CrossRef] [PubMed]

61. Oka, K.; Kinoshita, T.; Ise, A. Compact complete imaging polarimeter using birefringent wedge prisms . Opt. Express 2003, 11, 1510-1519. [CrossRef] [PubMed]

62. Oka, K.; Kinoshita, T.; Ise, A. Channeled spectropolarimeter using a wavelength scanning laser and a channeled spectroscopic polarization state generator. In Polarization Science and Remote Sensing V; International Society for Optical Engineering: Bellingham, WA, USA, 2011; Volume 8160.

63. Dubreuil, M.; Rivet, S.; Le Jeune, B.; Cariou, J. Snapshot Mueller matrix polarimeter by wavelength polarization coding. Opt. Express 2007, 15, 13660-13668. [CrossRef] [PubMed]

64. Dubreuil, M.; Tissier, F.; Le Roy, L.; Pennec, J.P.; Rivet, S.; Giroux-Metges, M.A.; Le Grand, Y. Polarization-resolved second harmonic microscopy of skeletal muscle in sepsis. Biomed. Opt. Express 2018, 9, 6350-6358. [CrossRef] [PubMed]

65. Le Gratiet, A.; Rivet, S.; Dubreuil, M.; Le Grand, Y. 100 kHz Mueller polarimeter in reflection configuration. Opt. Lett. 2015, 40, 645-648. [CrossRef]

66. Rivet, S.; Bradu, A.; Podoleanu, A. $70 \mathrm{kHz}$ full $4 \times 4$ Mueller polarimeter and simultaneous fiber calibration for endoscopic applications. Opt. Express 2015, 23, 23768-23786. [CrossRef]

67. Chue-Sang, J.; Bay, Y.; Stoff, S.; Gonzalez, M.; Holness, N.; Gomes, J.; Jung, R.; Gandjbakhche, A.; Chernomordik, V.; RamellaRoman, J. Use of Mueller matrix polarimetry and optical coherence tomography in the characterization of cervical collagen anisotropy. J. Biomed. Opt. 2017, 22, 1-9. [CrossRef] [PubMed]

68. Le Gratiet, A.; Pesce, L.; Oneto, M.; Marongiu, R.; Zanini, G.; Bianchini, P.; Diaspro, A. Circular intensity differential scattering (CIDS) scanning microscopy to image chromatin-DNA nuclear organization. OSA Contin. 2018, 1, 1068-1078. [CrossRef]

69. Marongiu, R.; Le Gratiet, A.; Pesce, L.; Bianchini, P.; Diaspro, A. ExCIDS: a combined approach coupling Expansion Microscopy (ExM) and Circular Intensity Differential Scattering (CIDS) for chromatin-DNA imaging. OSA Contin. 2020, 3, 1770-1780. [CrossRef]

70. Bueno, J.M.; Campbell, M.C.W. Confocal scanning laser ophthalmoscopy improvement by use of Mueller-matrix polarimetry. Opt. Lett. 2007, 27, 830-832. [CrossRef]

71. Alali, S.; Vitkin, A. Polarized light imaging in biomedicine: emerging Mueller matrix methodologies for bulk tissue assessment. J. Biomed. Opt. 2015, 20, 61104. [CrossRef]

72. Qi, J.; Elson, D. Mueller polarimetric imaging for surgical and diagnostic applications: a review. J. Biophotonics 2017, 10, 950-982. [CrossRef] 
73. He, H.; Liao, R.; Zeng, N.; Pengcheng, L.; Chen, Z.; Liu, X.; Hui, M. Mueller Matrix Polarimetry-An Emerging New Tool for Characterizing the Microstructural Feature of Complex Biological Specimen. J. Light. Technol. 2019, 37, 2534-2548. [CrossRef]

74. Dubreuil, M.; Babilotte, P.; Martin, L.; Sevrain, D.; Rivet, S.; Le Grand, Y.; Le Brun, G.; Turlin, B.; Le Jeune, B. Mueller matrix polarimetry for improved liver fibrosis diagnosis. Opt. Lett. 2012, 37, 1061-1063. [CrossRef] [PubMed]

75. Ben Hatit, S.; Foldyna, M.; De Martino, A.; Drévillon, B. Angle-resolved Mueller polarimeter using a microscope objective. Phys. Status Solidi (A) Appl. Mater. 2008, 205, 743-747. [CrossRef]

76. Arteaga, O.; Canillas, A. Measurement of the optical activity of anisotropic samples by transmission Mueller matrix ellipsometry. EPJ Web Conf. 2010, 5, 03001. [CrossRef]

77. Aas, L.; Foldyna, M.; De Martino, A.; Drévillon, B. Near infra-red mueller matrix imaging system and application to retardance imaging of strain. Journal of biomedical optics. Thin Solid Films 2011, 519, 2737-2741. [CrossRef]

78. Ossikovski, R.; De Martino, A. Differential Mueller matrix of a depolarizing homogeneous medium and its relation to the Mueller matrix logarithm. J. Opt. Soc. Am. A 2015, 32, 343-348. [CrossRef]

79. Sheppard, C.A.B.; Le Gratiet, A.; Diaspro, R. Coherency and differential Mueller matrices for polarizing media. J. Opt. Soc. Am. A 2018, 35, 2058-2069. [CrossRef] [PubMed] 